
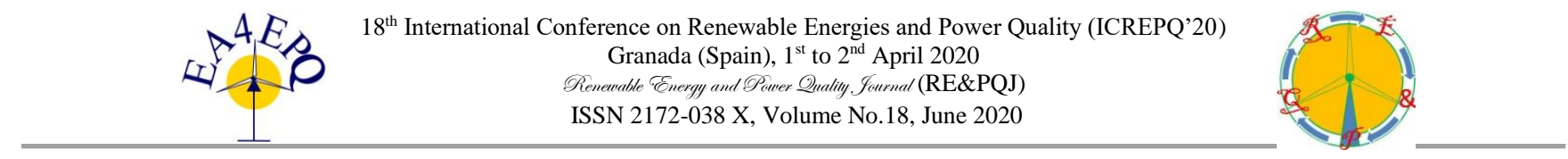

\title{
Understanding Resonance in a Renewable Energy Power Plant
}

\author{
David Scheepers and Johan Beukes \\ Department of Electrical Engineering \\ Stellenbosch University \\ Stellenbosch, 7600 (South Africa) \\ Phone number: +27 (83) 281-0529, e-mail: davidjjs@me.com
}

\begin{abstract}
This paper investigates the effect of various causes of resonance found in a typical renewable energy power plant. The consequences of resonance are discussed, and a simplified single-phase equivalent model of a plant is mathematically described and modelled in MATLAB. Reactive element interaction that causes resonant frequencies is discussed. Results obtained from the simplified mathematical model are compared with a detailed DIgSILENT PowerFactory model of a real installation. It is concluded that using the mathematical equations for the harmonic impedance profiles could provide insight into the expected resonant frequencies in a plant and possible increase in harmonic current propagation.
\end{abstract}

Key words. Harmonics, Resonance, Power Quality, Impedance Profile, Mathematical Modelling, Harmonic Interaction.

\section{Introduction}

The increasing introduction of renewable energy power plants (RPPs) around the world over the past two decades [1] encourages research into the effects of harmonics introduced by these RPPs. Harmonic assessment research is required both for utilities to apply sensible restrictions and for governing groups to establish proper regulations regarding harmonic limit compliance and assessment techniques.

The study of harmonics in power systems have been around since as early as the 19th century [2]. Harmonics are required to meet standards, such as established by the International Electrotechnical Commission (IEC) [3] or Institute of Electrical and Electronics Engineers (IEEE) [4], to verify whether it complies with network harmonic limit requirements. These restrictions are required as excessive harmonics could cause system malfunction at generation plants [8]. Harmonic resonance is, however, inevitable when connecting an RPP to the grid.

The introduced additional reactive elements interact with the grid to create or shift parallel and series resonant frequencies. These resonant frequencies could increase or reduce harmonic current emissions or voltage distortion levels at the point of connection (POC). Methods for determining whether harmonic currents are being absorbed or injected are discussed in [3] and [5] but considering specific causes of harmonic impedance changes could provide greater insight into the propagation of harmonic currents. Current post-connection methods for establishing the source of harmonics is also not always viable, since resonance is often ignored.

Various reactive and resistive elements contribute towards an installation's harmonic impedance. The components that introduce reactance are typically cables, transformers and capacitors. Component power losses does, however, introduce damping at resonant frequencies [10].

Developing a simplified mathematical model containing the required reactive elements, as done for a wind power plant in [9], whilst also using a mathematical model to identify reactive elements contributing towards specific resonances will be the focus of this paper. The simplified mathematical model is compared to a MATLAB Simulink model as well as a detailed PowerFactory model of an actual RPP.

\section{Simplified RPP model framework}

Considering the vast number of variables in a typical RPP mathematically modelling such a system in detail is considerably complex. To deal with this complexity certain simplifications and assumptions are made. By simplifying the circuit, the main contributors to specific harmonic frequencies can be identified and quantified.

A schematic diagram of a typical RPP installation is shown in Fig. 1. The POC is the bus where the installation is connected to the grid at high voltage, typically $132 \mathrm{kV}$. The first component in the installation is the station transformer, rated at the plant capacity, in this case 40 MVA. The station transformer provides a distribution cable network at a suitable medium voltage level of typically $22 \mathrm{kV}$. Field stations clusters various inverter systems together. These inverter systems contain medium to low voltage transformers and inverters. 
A lumped element representation of the typical system is derived by assessing components starting from the grid to the inverter.

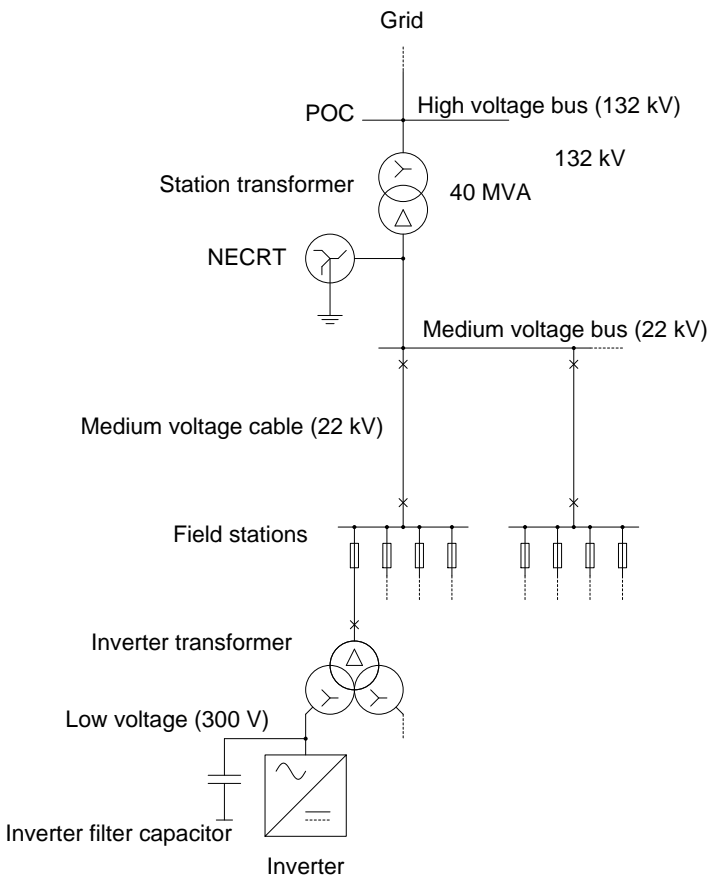

Fig. 1. Illustration of a typical RPP installation

\section{A. Grid impedance}

The network will be considered in its Thevenin equivalent form therefore, being represented as a voltage source and impedance in series with the rest of the system. The network impedance can be more complex when capacitive reactive components are present, but this paper focuses on internal resonances caused by an RPP. The grid impedance is defined as:

$$
Z_{s}=R_{s}+j X_{s}=R_{s}+j \omega L_{s}
$$

\section{B. Station transformer impedance}

Two-winding power transformers are modelled with both primary and secondary winding reactance and resistance as well as core reactance and resistance thus including core losses. Exciting current that induces core losses can be ignored because it only obtains a small percentage of the rated current (i.e. $<5 \%$ ) therefore, allowing the core reactance and resistance to be disregarded [6] Furthermore, the primary and secondary winding reactance and resistance can be lumped together. The station transformer impedance is defined as:

$$
Z_{s t}=R_{s t}+j X_{s t}=R_{s t}+j \omega L_{s t}
$$

\section{Medium voltage cable impedance}

Due to the close proximity of conductors in a cable, capacitive reactance can be significant to produce a low frequency resonant frequency. Since the grounded cable length is short (i.e. $<5 \mathrm{~km}$ ) a lumped circuit model is adequate. The cable inductive reactance is insignificant when compared to the transformer reactance in series with the cable and is therefore omitted. The cabling impedance is defined as a single capacitance:

$$
Z_{c}=j X_{c}=-j \frac{1}{\omega C_{c}}
$$

\section{Inverter transformer impedance}

The inverter transformer is modelled in the same way as the station transformer with impedance defined as:

$$
Z_{s}=R_{i t}+j X_{i t}=R_{i t}+j \omega L_{i t}
$$

\section{E. Inverter impedance}

In an inverter the output voltage is carefully controlled to control the output current through a filter inductor which reactance can therefore be neglected. The filter capacitor reactance is included, because it comes after the current source inverter equivalent circuit. Its impedance is defined as:

$$
Z_{i}=j X_{i}=-j \frac{1}{\omega C_{i}}
$$

The system in Fig. 1 now reduces to the lumped element representation in Fig. 2.

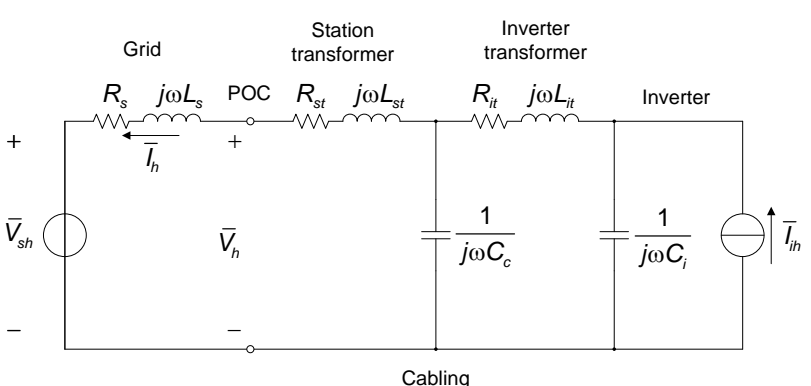

Fig. 2. Simplified lumped element single-phase equivalent model of an RPP installation

\section{Reactive element contribution to resonant frequencies}

The simplified lumped element single-phase equivalent model of an RPP installation is used to derive equations for the resonant frequencies and in the process the reactive elements taking part. To simplify the equations, the resistive elements were omitted, but are considered in Section 4.

From Fig. 2 a mathematical representation of the impedance at the POC can be derived. The first part of the equation consists of the inverter-side reactance $\left(j X_{s e r}\right)$ which includes the transformer inductance $\left(L_{i t}\right)$ in series with the inverter filter capacitor $\left(C_{i}\right)$.

$$
\begin{aligned}
j X_{\text {ser }} & =j \omega L_{i t}+\frac{1}{j \omega C_{i}} \\
& =\frac{1-\omega^{2} L_{i t} C_{i}}{j \omega C_{i}}
\end{aligned}
$$

The next combination consists of the cable capacitance $\left(C_{c}\right)$ in parallel with the inverter-side reactance $\left(j X_{s e r}\right)$. 


$$
\begin{aligned}
j X_{p a r}= & \frac{1}{\frac{1}{j X_{s e r}}+j \omega C_{c}} \\
& =\frac{1}{\frac{j \omega C_{i}}{1-\omega^{2} L_{i t} C_{i}}+j \omega C_{c}} \\
& =\frac{1-\omega^{2} L_{i t} C_{i}}{j \omega C_{i}+j \omega C_{c}\left(1-\omega^{2} L_{i t} C_{i}\right)} \\
& =\frac{1-\omega^{2} L_{i t} C_{i}}{j \omega\left(C_{i}+C_{c}\right)\left[1-\omega^{2} L_{i t} \frac{C_{i} C_{c}}{C_{i}+C_{c}}\right]}
\end{aligned}
$$

Equation (8), representing the reactance as seen at the POC, is derived from the parallel combination of the network reactance $\left(L_{s}\right)$ and the series combination of the station transformer reactance $\left(j \omega L_{s t}\right)$ and the installation side reactance $\left(j X_{p a r}\right)$. This equation is acquired through some mathematical manipulation.

$$
\begin{aligned}
& j X_{P O C}=\frac{1}{\frac{1}{j \omega L_{s t}+j X_{p a r}}+\frac{1}{j \omega L_{s}}} \\
& =\frac{1}{\frac{1}{1-\omega^{2} L_{i t} C_{i}}}+\frac{1}{j \omega L_{s}} \\
& \left.=j \omega L_{s t}+\frac{j \omega\left(C_{i}+C_{c}\right)\left[1-\omega^{2} L_{i t} \frac{C_{i} C_{c}}{C_{i}+C_{c}}\right]}{1-\omega^{2}\left[\left(L_{s}+L_{s t}+L_{i t}\right) C_{i}+\left(L_{s}+L_{s t}\right) C_{c}\left(1-\omega^{2} L_{i t} C_{i}\right)\right]} C_{i}+L_{i t} C_{c}\left(1-\omega^{2} L_{i t} C_{i}\right)\right]
\end{aligned}
$$

From equation (8) various terms can be identified that contribute to either series resonance or parallel resonance. These terms are evaluated according to their frequency dominance and are discussed subsequently.

\section{A. Low resonant frequencies}

The first resonant frequency occurs due to the interaction of the series combination of all four inductive elements in series with the inverter filter capacitor, as illustrated in Fig. 3 below. The assessment position is outside the current loop and parallel resonance is experienced.

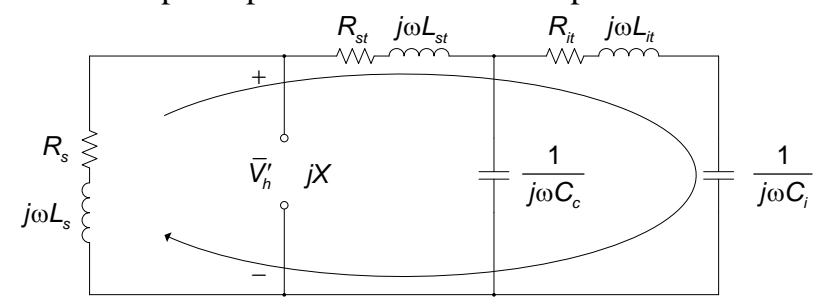

Fig. 3. Assessment loop of elements forming part of the low order parallel resonance reactance

The resonant frequency is calculated using the denominator of (8). For lower frequencies the second term in the denominator is insignificantly small relative to the first term. Ignoring the second term will, however, introduce a slight error in identifying the exact resonant frequency but is considered insignificant. The following equation is derived for calculating the first resonant frequency.

$$
\begin{aligned}
& 1-\omega_{1}^{2}\left[\left(L_{s}+L_{s t}+L_{i t}\right) C_{i}+\left(L_{s}+L_{s t}\right) C_{c}\left(1-\omega^{2} L_{i t} C_{i}\right)\right]=0 \\
& \Rightarrow 1-\omega_{1}^{2}\left(L_{s}+L_{s t}+L_{i t}\right) C_{i} \approx 0 \\
& \Rightarrow \omega_{1} \approx \frac{1}{\sqrt{\left(L_{s}+L_{s t}+L_{i t}\right) C_{i}}}
\end{aligned}
$$

The second distinctive resonant frequency occurs due to the transformer and inverter-side reactance in series with the inverter filter capacitance, as illustrated in Fig. 4 below. The position of assessment is part of the assessment loop that is formed by these elements, therefore, causing series resonance.

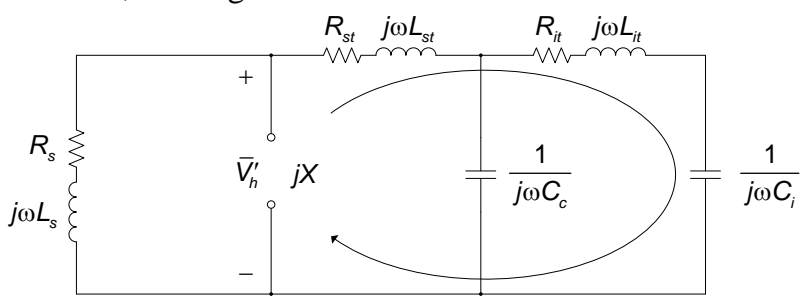

Fig. 4. Assessment loop of elements forming part of the low order series resonance reactance

The resonant frequency for this case is calculated using the numerator of equation (8). The second resonance is calculated as follows.

$$
\begin{aligned}
& 1-\omega_{2}^{2}\left[\left(L_{s t}+L_{i t}\right) C_{i}+L_{s t} C_{c}\left(1-\omega_{2}^{2} L_{i t} C_{i}\right)\right]=0 \\
& \Rightarrow 1-\omega_{2}^{2}\left(L_{s t}+L_{i t}\right) C_{i} \approx 0 \\
& \Rightarrow \omega_{2} \approx \frac{1}{\sqrt{\left(L_{s t}+L_{i t}\right) C_{i}}}
\end{aligned}
$$

What is notable here is the difference between these two frequencies is determined by the value of line inductance. The weaker the network the lower the parallel resonant frequency will move away from the series resonant frequency on the spectrum.

\section{B. High resonant frequencies}

The third and fourth resonant frequencies occur higher on the frequency spectrum than the previously discussed resonant frequencies. At higher frequencies the inverter transformer inductive reactance increases to such an extent that the cable capacitor becomes significant. This also complicates solving the equation to determine these parallel and series resonant frequencies, since all the terms in (8) becomes imperative. The remaining resonance assessment loops are illustrated as seen in Fig. 5 a) and b) below. 

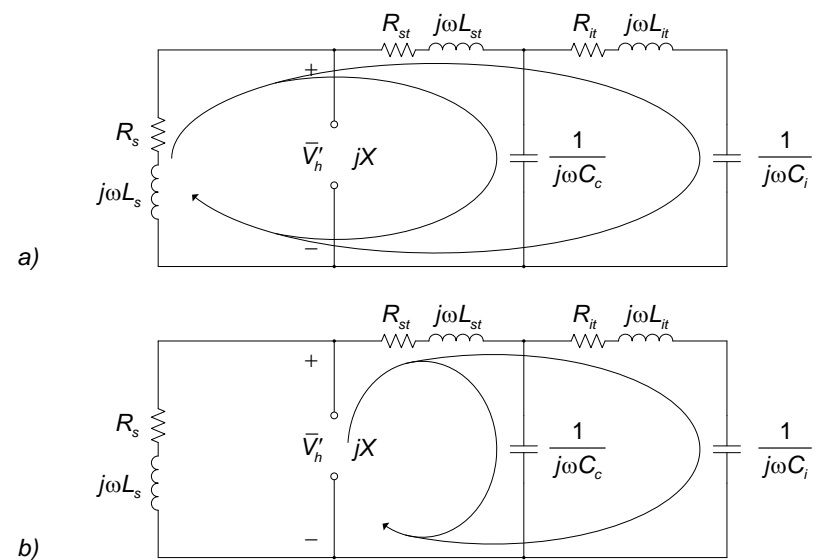

Fig. 5. Assessment loop of elements forming part of the higher order a) parallel and b) series resonance reactance respectively

Due to the simplification methods that were used previously not being applicable the second roots for both the denominator and the numerator are calculated using their $4^{\text {th }}$ degree polynomials. This can is achieved through an iterative process such as the Newton-Raphson method [7].

\section{Simulation results and comparisons}

The model as depicted in Fig. 2 was developed accordingly in MATLAB Simulink to determine whether the mathematical model represents the physical model. Various resistors were also included with each reactive element to introduce damping.

A detailed PowerFactory model of an RPP was also developed to which the mathematical and Simulink model can be compared to identify the accuracy.

Parameters defined for the simplified model are subsequently derived from the PowerFactory model.

\section{A. Grid impedance}

In Powerfactory the grid is modelled by an equivalent voltage source with source impedance values as follows:

$$
\begin{aligned}
& R_{s}=8.39 \Omega \\
& \mathrm{X}_{s}=21.7 \Omega \\
& \Rightarrow L_{s}=\frac{21.7}{100 \pi}=69 \mathrm{mH}
\end{aligned}
$$

\section{B. Station transformer impedance}

The station transformer has a rating of $40 \mathrm{MVA}$ and at $132 \mathrm{kV}$ the rated current is calculated as follows:

$$
I_{s t}=\frac{40 \cdot 10^{6}}{\sqrt{3} \times 132 \cdot 10^{3}}=175 \mathrm{~A}
$$

The transformer has an impedance of $11.5 \%$ and the voltage drop is, therefore,

$$
V_{11.5 \%}=0.115 \times \frac{132 \cdot 10^{3}}{\sqrt{3}}=8760 \mathrm{~V}
$$

The transformer impedance is calculated as follows:

$$
Z_{s t}=\frac{V_{11.5 \%}}{I_{s t}}=\frac{8760}{175}=50 \Omega
$$

The transformer total copper loss is $200 \mathrm{~kW}$, or $66.7 \mathrm{~kW}$ per phase, that means the equivalent series resistance is:

$$
R_{s t}=\frac{P_{t}}{I_{b}{ }^{2}}=\frac{66.7 \cdot 10^{3}}{175^{2}}=2.177 \Omega
$$

The transformer inductance can now be calculated as follows:

$$
\begin{aligned}
& X_{t}=\sqrt{Z_{t}-R_{t}}=\sqrt{50^{2}-2.177^{2}}=49.95 \Omega \\
& \Rightarrow L_{s t}=\frac{X_{t}}{\omega}=\frac{49.95}{100 \pi}=159 \mathrm{mH}
\end{aligned}
$$

\section{Medium voltage cable impedance}

From the station transformer medium voltage cable spur out to 6 field stations that feed 30 inverter containers. The average cable length to inverter containers is $1.5 \mathrm{~km}$ and they have capacitive reactance of approximately $0.192 \mu \mathrm{F} / \mathrm{km}$. An approximation of the cable capacitance transformed to the $132 \mathrm{kV}$ side can, therefore, be done as follows:

$$
C_{c}=30 \times 1.5 \times 0.192 \cdot 10^{-6} \times \frac{22^{2}}{132^{2}}=240 \mathrm{nF}
$$

\section{Inverter transformer impedance}

In terms of significant inductive reactance, the next element represents the inverter transformers connecting the low voltage $(300 \mathrm{~V})$ inverters to the medium voltage $(22 \mathrm{kV})$ cabling.

A three winding transformer is employed and each secondary winding has a capacity of $725 \mathrm{kVA}$. The rated current per secondary winding transformed to the medium voltage side is calculated as follows:

$$
I_{i t}=\frac{0.725 \cdot 10^{6}}{\sqrt{3} \times 22 \cdot 10^{3}}=19 \mathrm{~A}
$$

The inverter transformer has an impedance of $5.59 \%$. At rated current the voltage drop is, therefore,

$$
V_{5.59 \%}=0.059 \times \frac{22 \cdot 10^{3}}{\sqrt{3}}=710 \mathrm{~V}
$$

The transformer impedance is calculated as follows:

$$
Z_{i t 1}=\frac{V_{5.59 \%}}{I_{i t}}=\frac{710}{19}=21.5 \Omega
$$

The transformer copper loss is $6.5 \mathrm{~kW}$, or $2.16 \mathrm{~kW}$ per phase that means the equivalent series resistance is:

$$
R_{i t 1}=\frac{P_{i t}}{I_{i t}{ }^{2}}=\frac{1}{3} \times \frac{6500}{19^{2}}=6 \Omega
$$

The transformer inductance can now be calculated as follows:

$$
\begin{aligned}
& X_{i t 1}=\sqrt{Z_{i t 1}{ }^{2}-R_{i t 1}{ }^{2}}=\sqrt{21.5^{2}-3.46^{2}}=21.3 \Omega \\
& \Rightarrow L_{i t 1}=\frac{X_{i t 1}}{\omega}=\frac{21.3}{100 \pi}=67.7 \mathrm{mH}
\end{aligned}
$$

60 transformer windings are lumped together and transformed to $132 \mathrm{kV}$ to obtain an equivalent inverter transformer impedance as follows: 


$$
\begin{aligned}
& L_{i t}=\frac{1}{60} \times \frac{132^{2}}{22^{2}} \times 0.0677=40.6 \mathrm{mH} \\
& \mathrm{R}_{i t}=\frac{1}{60} \times \frac{132^{2}}{22^{2}} \times 3.46=2.07 \Omega
\end{aligned}
$$

\section{E. Inverter filter capacitance}

Each of the 60 inverters operates at $300 \mathrm{~V}$ and has $400 \mu \mathrm{F}$ capacitors connected in delta in its low pass filter. The lumped capacitive reactance in the equivalent $132 \mathrm{kV}$ model converted to phase values is calculated as follows:

$$
C_{i}=3 \times 60 \times \frac{0.3^{2}}{132^{2}} \times 400 \cdot 10^{-6}=0.372 \mu \mathrm{F}
$$

The list detailing the various parameter values, as transformed to the HV equivalent circuit, can be seen in Table I.

Table I: Parameters for the Simulink model as calculated from PowerFactory model

\begin{tabular}{|l|l|}
\hline Installation parameter & Value \\
\hline Network inductance $\left(L_{s}\right)$ & $69 \mathrm{mH}$ \\
\hline Network resistance $\left(R_{s}\right)$ & $8.39 \Omega$ \\
\hline Station transformer inductance $\left(L_{s t}\right)$ & $159 \mathrm{mH}$ \\
\hline Station transformer resistance $\left(R_{s t}\right)$ & $2.177 \Omega$ \\
\hline Cable capacitance $\left(C_{c}\right)$ & $240 \mathrm{nF}$ \\
\hline Inverter transformer inductance $\left(L_{i t}\right)$ & $40.6 \mathrm{mH}$ \\
\hline Inverter transformer resistance $\left(R_{i t}\right)$ & $2.07 \Omega$ \\
\hline Inverter filter capacitance $\left(C_{i}\right)$ & $372 \mathrm{nF}$ \\
\hline
\end{tabular}

The approximate resonant frequencies as suggested by (8) is calculated using (9) and (10) with the higher order resonant frequencies being calculated through applying the Newton-Raphson method. The results are documented in Table II.

Table II: Resonant frequencies calculated from the mathematical model.

\begin{tabular}{|l|l|}
\hline $\begin{array}{l}\text { Resonant } \\
\text { frequency }\end{array}$ & Frequency \\
\hline$\omega_{1}$ & $504 \mathrm{~Hz}$ \\
\hline$\omega_{2}$ & $584 \mathrm{~Hz}$ \\
\hline$\omega_{3}$ & $2137 \mathrm{~Hz}$ \\
\hline$\omega_{4}$ & $2169 \mathrm{~Hz}$ \\
\hline
\end{tabular}

When comparing with the Simulink model the results are verified as resonance occurs at approximately the same frequencies as determined in Table II, this is indicated in Fig. 6. The error in lower order resonance is due to the small difference in capacitance between the inverter filter capacitance and the cable capacitance. The original simplification ignored the cable capacitance's effect leading to a $\pm 100 \mathrm{~Hz}$ difference.
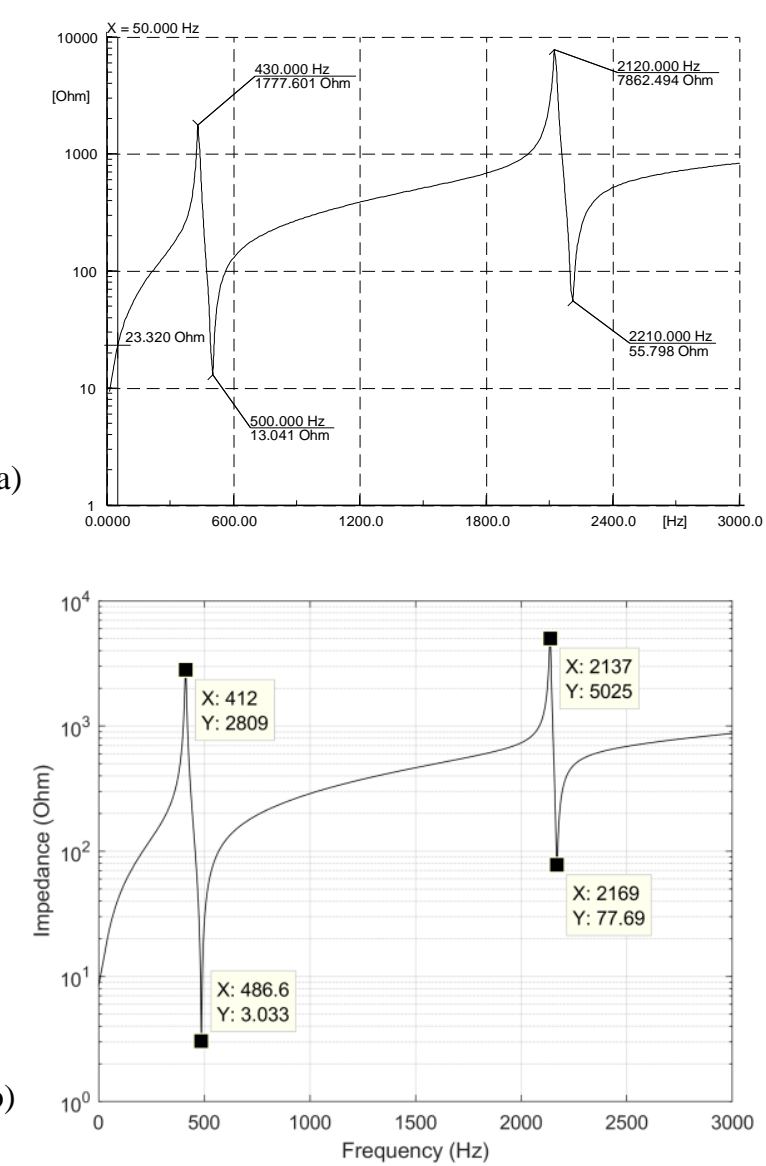

Fig. 6. a) PowerFactory and b) Simulink impedance profile at the POC of a modelled RPP system.

The harmonic impedance of the simplified single-phase equivalent model is significantly similar to the detailed PowerFactory model. This illustrates the accuracy of the developed mathematical approach.

\section{Conclusion}

A mathematical model used to represent an RPP was developed and analysed to determine which components significantly contribute towards specific points of resonance. The interaction between reactive elements were considered to determine the frequencies of resonance, ignoring resistance which mostly contributes towards damping at the resonant frequencies.

The results of the mathematical model were compared to a frequency impedance sweep executed at the POC of a Simulink model, which included resistive components as stated in Table I. The results were compared with a PowerFactory model which validated the results.

It was determined that the mathematical model successfully represents the developed Simulink model and that the suggested mathematical method of determining resonant frequencies is quite accurate in approximating the respective points of resonance. Parameter uncertainty caused a slight variation of the resonant frequencies.

The practicality of investigating the harmonic impedance to determine the likely source of harmonics is concluded. Network variability with regards to existing resonance 
within the connected network was disregarded for this study as existing resonance would occur at higher frequencies.

This paper evaluated the harmonic impedance of a fully operating RPP. The RPP could, however, experience significant transient impedance changes during different conditions causing resonance to shift. The effect of these transitions would therefore still need to be quantified.

\section{References}

[1] F. Dinçer, "The analysis on photovoltaic electricity generation status, potential and policies of the leading countries in solar energy," Renewable and Sustainable Energy Reviews, vol. XV, no. 1, pp. 713-720, 2011.

[2] A. E. Emanuel, "Harmonics in the early years of electrical engineering: a brief review of event, people and documents," in Ninth International Conference on Harmonics and Quality of Power. Proceedings (Cat. No.00EX441), Orlando, FL, USA, 2000.
[3] IEC TR Standard 61000-3-6, Electromagnetic compatibility (EMC) - Part 3-6: Limits - Assessment of emission limits for the connection of distorting installations to $M V, H V$ and EHV power systems, 2008.

[4] IEEE Recommended Practice and Requirements for Harmonic Control, Electric Power System IEEE Standard 519-2014, 2014.

[5] CIGRE WG C4/B4.38, Network Modelling for Harmonic Studies, 2017.

[6] J. D. Glover, M. S. Sarma and T. J. Overbye, Power system analysis and design fifth edition, Cengage Learning, 2010.

[7] T. J. Ypma, "Historical Development of the NewtonRaphson Method," SIAM Review, vol. XXXVII, no. 4, pp. 531-551, 1995.

[8] J. Arrilaga and N. Watson, Power system harmonics, John Wiley \& Sons, 2004.

[9] K. Hasan, K. Rauma, A. Luna, J. Candela and P. Rodriguez, "Harmonic Resonance Study for Wind Power Plant," $R E \& P Q J$, vol. 1, no. 10, pp. 1507-1512, 2012.

[10] W. Xu, Z. Huang, Y. Cui and H. Wang, "Harmonic resonance mode analysis," IEEE Transactions on Power Delivery, vol. 20, no. 2, pp. 1182-1190, 2005. 